\title{
Instantaneous Respiratory Rate Estimation from multilead ECG delineation using VCG directions on fiducial points
}

\author{
Maikel Noriega ${ }^{1}$, Ennis Carcases, K. Duran, Enrique Juan Marañón, Juan Pablo Martínez ${ }^{2,3}$, Rute \\ Almeida $^{3,4}$ \\ ${ }^{1}$ Communications and Electronical Departament of Oriente University, Santiago de Cuba (Cuba) \\ ${ }^{2}$ Aragón Institute of Engineering Research (I3A), University of Zaragoza, Aragón, Spain \\ ${ }^{3}$ BSICoS Group, CIBER-BBN, Spain. \\ ${ }^{4}$ CMUP - Centro de Matemática da Universidade do Porto, FCUP, Porto, Portugal.
}

\begin{abstract}
The main purpose of this work is the estimation of the respiratory rate $\left(r_{R}\right)$ from the electrocardiogram (ECG). In this study, the $r_{R}$ was estimated from the same Fit directions of maximum projection (FD) used for multi lead ECG automatic delineation (ML). A previously developed and validated methodology for boundaries location was extended to include wave peaks and estimate FD. The median of power spectral density obtained over the directions based on QRS complex main peak, $T$ wave peak and end spatial loops was used for $r_{R}$ estimation. In a control database, the proposed method yielded more accurate $r_{R}$ estimates (mean absolute error (MAE), 2.64 bpm, $S D=3.92)$ than the estimates based on the single-lead ECG $R$-peak amplitude (MAE values from 3.29 to $5.26 \mathrm{bpm}, S D$ $\geq 5$ ) and RR series (2.89 to $3.66 \mathrm{bpm}, S D>4.6$ ), close to results from EDR method (2.89 bpm, $S D=3.63$ ).
\end{abstract}

\section{Introduction}

Direct estimation of respiratory rate $\left(r_{R}\right)$ is done with devices that are intrusive, expensive and uncomfortable for the patient [1]. Therefore the development of alternative methods for indirect $r_{R}$ estimation is of great interest. The respiratory and heart activities are linked through physiological processes. The respiration modulates the heart rate such that it increases during inspiration and decreases during expiration [2]. In the same way, during the respiratory cycle, chest movements and changes in the thorax impedance distribution due to filling and emptying of the lungs cause a rotation of the electrical axis of the heart which has an effect over beat morphology [3,4]. Different signal processing techniques have been developed to extract respiratory information from the modulation of the ECG R-peak amplitudes (RPA) and heart rate (HR) [4-8]. However, the single-lead RPA and RR series have been shown not to yield accurate $r_{R}$ estimates as the respiratory influence in a given ECG lead is subject dependent [4]. Often, in ambulatory and clinical applications, multilead ECG recordings are available, allowing to construct vectocardiographic (VCG) loops from orthogonal leads. In [4] the series of rotation angles of the heart's electrical axis as induced by respiration are estimated from least-squares loop alignment, and the $r_{R}$ estimated spectrally (EDR). The main directions on the wavelet transform (WT) of VCG loops, taken as parallel to each ECG fiducial point, have been successfully used for multilead delineation of wave boundaries [9]. Moreover a strong relation between the respiration and the $T$ wave end based Fit directions of maximum projection (FD) used the by multi lead ECG delineation system (ML) was found in [10]. Taking advantage of this relation, in [11] we have been able to estimate $r_{R}$ with good results from the FD used for T wave end delineation. In this study we aim to combine the information based in FD found for the delineation of QRS peak and T wave peak and end to obtain a single $r_{R}$ estimation.

\section{Methods}

\subsection{Data}

The data used in this study was a database of ECG signals from healthy subjects recorded at University of Zaragoza, which we call control database (CDB). This database contains the standard 12-lead ECG and a respiration signal of 40 subjects (age $32 \pm 9$ years, 26 male / 14 female), recorded during 5 minutes of supine resting and all were here considered. The MP 150 (BIOPAC Systems) was used to acquire simultaneously the ECG (ECG100C amplifier and disposable $\mathrm{Ag}-\mathrm{AgCl}$ electrodes) at a sampling frequency of $1000 \mathrm{~Hz}$, and the respiration signal (RSP100C and a strain gauge transducer) at a sampling frequency of $125 \mathrm{~Hz}$. 


\subsection{Multilead ECG delineation}

The ML delineation system considered was previously proposed and validated in [9] and is included in BioSigBrowser, a user-friendly Matlab interface [12]. It uses two or three orthogonal leads of the ECG to define spacial vectocardiographic loops (VCG). Denoting the WT of a signal $s(n) \in\{x(n), y(n), z(n)\}$ at scale $m$ by $w_{s, m}[n]$, the spatial WT loop is defined as:

$$
\mathbf{w}_{m}(n)=\left[w_{x, m}(n), w_{y, m}(n), w_{z, m}(n)\right]^{T}
$$

As a consequence of the WT prototype used, the WT loop $\left.\mathbf{w}_{m}(n)\right|_{n \in L}$ is proportional to the VCG derivative and describes the velocity of evolution of the electric heart vector (EHV) in a time interval $L$. The main direction $\mathbf{u}=\left[u_{x}, u_{y}, u_{z}\right]^{T}$ of EHV variations on any time interval $L$ is given by the director vector of the best straight linear fit to all points in $w_{m}(n)$ and is here called Fit direction of maximum projection (FD). The FD can be interpreted as corresponding to the ECG lead maximizing the local SNR, and thus, it is the most appropriate for delineation purposes [9]. The projection of $\mathbf{w}_{m}(n)$ over the direction $\mathbf{u}$ allows to obtain a derived wavelet signal $w_{d, m}(n)$ that combines the information provided by the 3 or 2 available leads:

$$
w_{d, m}(n)=\frac{\mathbf{W}_{m}^{T}(n) \cdot \mathbf{u}}{\|\mathbf{u}\|} ; n \in I
$$

The time intervals $L$ (used for linear fitting) and $I$ (used for projecting) can be different, depending on each wave specificities. The strategy proposed for ML boundary location using WT loops is based in a multi-step iterative search for a better spatial lead (with steeper slopes) for delineation. The goal is to construct a derived wavelet signal well suited for boundaries location, using the same detection criteria as in the SL delineator proposed in [13]. The ML system was originally designed for boundaries detection only, with the ML peak positions defined as the median of the SL peak positions. This method is very simple, but represents the main weakness of the ML system as it is very sensitive to the SL measurements on the original leads, and does not have a clear physiological interpretation. In this work, we extend the method for ML delineation of wave peaks, as explained below.

A) New ML wave peak delineation. The strategy is similar to the one for ML wave boundaries [9], with the specificities described in the following general algorithm (particular details for each wave peak are described in later sections). For each beat k:

\section{Step 1 - initialization}

$\left.a_{1}\right)$ an initial search window adequate to find the EHV main direction in the peak is defined as $W^{[1]}$; $\left.b_{1}\right)$ the initial main direction of EHV variations $\mathbf{u}^{[1]}$ is estimated as the best line fit in total least squares (TLS) sense [14] to $\left.\mathbf{w}_{m}(n)\right|_{n \in W^{[1]}}$, using the adequate scale $2^{m}$;

$\left.c_{1}\right)$ the loop $\left.\mathbf{w}_{m}(n)\right|_{n \in I}$ is projected over $\mathbf{u}^{[1]}$ to construct the new derived WT signal $w_{d, m}^{[1]}(n)$;

$\left.d_{1}\right)$ SL delineation is performed over $w_{d, m}^{[1]}(n)$ to locate the peak position at step 1 .

\section{Step $\mathbf{i} \geq 2$ - iteration}

$\left.a_{\mathbf{i}}\right)$ the search window $W^{[\mathbf{i}]}$ is updated attending to the peak location provided by at step [i-1]

$\left.b_{\mathbf{i}}\right)$ the main direction of EHV variations $\mathbf{u}^{[\mathbf{i}]}$ is estimated as the TLS best line fit to $\left.\mathbf{w}_{m}(n)\right|_{n \in W^{[\mathbf{i}]}}$;

$\left.c_{\mathbf{i}}\right)$ the new derived WT signal $w_{d, m}^{[\mathbf{i}]}(n)$ is constructed by projecting the loop $\left.\mathbf{w}_{m}(n)\right|_{n \in I}$;

$\left.d_{\mathbf{i}}\right)$ IF no significant maximum of $w_{d, m}^{[\mathbf{i}]}(n)$ was found OR the amplitude of the maximum $n_{p}^{[i]}$ is equal or lower that the amplitude of $n_{p}^{[\mathbf{i}-1]}$ (found in the previous step) THEN $w_{d, m}^{[i]}(n)$ is less fitted for peak location than $w_{d, m}^{[\mathbf{i}-1]}(n)$ and the peak position at step [i-1] is adopted as ML mark; STOP;

ELSE SL delineation of the boundary is performed over $w_{d, m}^{[\mathbf{i}]}(n)$ to find the peak position updated boundary location;

$\left.e_{\mathbf{i}}\right)$ IF no relevant change is found in the boundary location THEN the location at step [i] is adopted as ML mark; STOP;

ELSE REPEAT from $a_{\mathrm{i}}$ ), for $i+1$.

$B)$ Specific parameters for $\mathbf{Q R S}$ complex peak. In the new ML system the location of the complex QRS main wave is determined in the WT loop in scale $2^{2}$. Noise or QRS wave morphology bad identification can produce outliers if FD chosen at step 1 does not corresponds to the higher modulus value. To avoid that, the search window length is reduced depending on the sampling frequency up to $40 \%$ of number of points. Let define $n_{Q R S, f}^{[0]}\left[n_{Q R S, l}^{[0]}\right]$ as the earliest [latest] significant maximum modulus location given by the SL methods (over each orthogonal lead). The initial search window for the peak of QRS main wave is taken as:

$$
W_{Q R S}^{[1]}=\left[n_{Q R S, f}^{[0]}+v ; n_{Q R S, l}^{[0]}-v\right]
$$

where $v$ is the $1 / 2$ reduction in number of samples with respect to the maximum length search window. The best line fine is estimated for each $v=0$ to $20 \%$ of $\left[n_{Q R S, f}^{[0]} ; n_{Q R S, l}^{[0]}\right]$ length and the initial main direction of EHV variations $u_{[1]}$ is taken as the one with maximum modulus. This ensures the higher signal-to-noise ratio in 
the projected derivation. At each iteration [i], the search window is updated as

$$
W_{Q R S}^{[\mathrm{i}]}=\left[n_{Q R S, f}^{[i-1]} ; n_{Q R S, l}^{[i-1]}\right]
$$

where $n_{Q R S, f}^{[\mathbf{i}-1]}\left[n_{Q R S, l}^{[\mathbf{i}-1]}\right]$ is the location of the first [last] significant maximum modulus of $w_{d, m}^{[\mathbf{i}-1]}(n)$. If $n_{Q R S, f}^{[\mathbf{i}]}\left[n_{Q R S, l}^{[\mathbf{i}]}\right]$ has the same polarity as $n_{Q R S, f}^{[\mathbf{i}-1]}\left[n_{Q R S, l}^{[\mathbf{i}-1]}\right]$ and equal or lower amplitude it is considered that the lead constructed at step [i] is not better for the QRS main wave peak location than the constructed in the step [i-1] and the iteration STOP criteria of $d_{\mathbf{i}}$ ) is applied. The iteration STOP criteria of $e_{\mathbf{i}}$ ) is applied if the the same location is achieved for 3 iterations.

C) Specific parameters for $\mathbf{T}$ wave peak. The location of the T wave is determined in the WT loop in scale $2^{5}$ if in the SL delineation scale $2^{5}$ was used for T wave detection for at least two out of the three leads, and $2^{4}$ otherwise. We defined the initial search window for the $\mathrm{T}$ wave peak as:

$$
W_{T}^{[1]}=\left[n_{T, f}^{[0]} n_{T, l}^{[0]}\right]
$$

where $n_{T, f}^{[0]}\left[n_{T, l}^{[0]}\right]$ is the location of the first [last] significant maximum modulus location given by the SL methods (over each orthogonal lead). At each iteration [i], the search window is updated to

$$
W_{T}^{[\mathbf{i}]}\left[n_{T, f}^{[\mathbf{i}-1]} n_{T, l}^{[\mathbf{i}-1]}\right]
$$

where $n_{T, f}^{[\mathbf{i}-1]}\left[n_{T, l}^{[\mathbf{i}-1]}\right]$ is the location of the first [last] significant maximum modulus of $w_{d, m}^{[\mathbf{i}-1]}(n)$.

\subsection{Estimating $r_{R}$}

The ECG signals of CDB were delineated using the ML delineation strategy over the Frank leads synthesized using the inverse Dower transformation [15]. From this delineation are obtained the vectors defining FD for each of heartbeat and fiducial points QRS peak, T wave peak and end, corresponding to the directions of interest $\left(\mathbf{u}_{h}^{[\mathbf{i}]}\right.$, where $h \in\{R p, T p, T e\}$, respectively). The coordinates of each $\mathbf{u}$, originally with one value per heart beat, were resampled uniformly at $2 \mathrm{~Hz}$ using cubic spline interpolation. The $r_{R}$ estimation is performed spectrally from the interpolated directions $\mathbf{u}_{h}^{[\mathbf{i}]}$. Estimation of the power spectrum is accomplished with Burg's method [16]. The spectrum of each directions series $(\mathbf{U}(f))$ is estimated with non overlapping windows of $60 \mathrm{~s}$ duration. The median of the individual running power spectra of all directions
$\left(\mathbf{U}_{m}(f)\right)$ is calculated in order to reduce the frequential peaks not related to breathing and to obtain a single $r_{R}$ estimation The global respiratory frequency was estimated on the median spectrum for each window $\left(f_{R}\right)$. Estimation of the respiratory frequency as the largest peak of $U_{m}(f)$ comes with the risk of choosing the location of a spurious peak. This risk is, however, considerably reduced by narrowing down the search interval to only include frequencies between $0.15 \mathrm{~Hz}$ and $0.45 \mathrm{~Hz}$ of respiratory frequency. The estimated $r_{R}$ in breaths-per-minute (bpm) is calculated from the respiratory frequencies. As a reference to evaluate the estimation of $r_{R}$ from FD, we estimated the $r_{R}$ from the respiratory impedance waveform. The respiratory impedance waveform was first resampled uniformly at $2 \mathrm{~Hz}$ using cubic spline interpolation, and band-pass filtered between $0.1 \mathrm{~Hz}$ and $0.5 \mathrm{~Hz}$. Then, the Burg's method was applied to obtain the $r_{R} r e f$. The accuracy of the ECG-based $r_{R}$ estimates was evaluated by averaging their values in 60-s windows and computing their mean absolute error $\left(M A E_{u}\right)$ and the standard deviation $\left(S D_{u}\right)$ of MAE in terms of breaths-per-minute with respect to the $r_{R}$ ref. Additionally, for the sake of comparison, $r_{R}$ was estimated from the single-lead RPA waveform and RR series obtained from SL delineation of CDB and using the EDR method. The RPA, RR and EDR rotation angles series were re-sampled uniformly at $2 \mathrm{~Hz}$ using cubic spline interpolation. The $r_{R}^{\text {lead }} R P A$ and $r_{R}^{\text {lead }} R R$ for each standard lead, and $r_{R}^{\text {lead }} E D R$ were estimated spectrally using the Burg's method with non-overlapping windows of 60 $\mathrm{s}$ duration, considering the median spectra of the 3 rotation angles for EDR. As before, the accuracy of estimation was evaluated by the MAE in terms of breaths-per-minute with respect to the $r_{R}$ ref and its $\mathrm{SD}\left(M A E_{s}^{\text {lead }} \pm S D_{s}^{\text {lead }}\right.$, $\left.s \in\{R P A, R R\}, M A E_{E D R} \pm S D_{E D R}\right)$.

\section{Results}

Table I reports the $M A E_{u} \pm S D_{u}$ across files estimated with the proposed method and, for the sake of comparison, the $M A E_{s} \pm S D_{s}$ values obtained for the RPA, RR based and EDR methods. Both the mean errors and $\mathrm{SD}$ in the estimation by proposed method are lower than any from single-lead RPA and RR based estimations, indicating lower bias and higher stability. Observe also that the uncertainty of the lead choice is eliminated with the proposed method. The best results are obtained by EDR method.

\section{Discussion and Conclusion}

The results obtained in this study show that it is possible to extract accurately the $r_{R}$ from the combination of directions $\mathrm{u}$, obtained from the ML delineator in the QRS peak and $\mathrm{T}$ wave peak and end. Additionally a new algorithm 
Table 1. Average error and standard deviation across files in BPM obtained from single-lead RPA and RR signals.

\begin{tabular}{ccc}
\hline \hline$M A E_{u} \pm S D_{u}$ (Proposed method) & $2.64 \pm 3.92$ \\
\hline \hline Lead & $M A E_{R P A}^{\text {Lead }} \pm S D_{R P A}^{\text {Lead }}$ & $M A E_{R R}^{\text {Lead }} \pm S D_{R R}^{\text {Lead }}$ \\
\hline V1 & $3.32 \pm 5.00$ & $3.20 \pm 5.23$ \\
V2 & $3.41 \pm 5.13$ & $3.04 \pm 5.02$ \\
V3 & $3.82 \pm 5.71$ & $2.99 \pm 4.63$ \\
V4 & $3.29 \pm 5.31$ & $3.28 \pm 5.33$ \\
V5 & $3.76 \pm 5.82$ & $2.92 \pm 4.87$ \\
V6 & $3.52 \pm 5.16$ & $2.89 \pm 4.86$ \\
I & $4.35 \pm 5.69$ & $3.43 \pm 5.58$ \\
II & $4.25 \pm 5.68$ & $3.21 \pm 5.16$ \\
III & $3.90 \pm 5.41$ & $3.32 \pm 5.02$ \\
aVR & $3.99 \pm 5.53$ & $3.32 \pm 5.02$ \\
aVL & $4.16 \pm 5.62$ & $3.66 \pm 5.04$ \\
aVF & $5.26 \pm 6.82$ & $3.42 \pm 5.52$ \\
\hline \multicolumn{2}{c}{$M A E_{E D R} \pm S D_{E D R}$} & $2.12 \pm 3.63$ \\
\hline \hline
\end{tabular}

of ML delineation is proposed for the QRS complex main wave and $\mathrm{T}$ wave peaks. The use of a multilead method not only eliminates the variability across leads, but also increases the stability of estimation, outperforming the single lead based methods. Thus, the beat-to-beat estimation of respiratory rate can be obtained as an extra output of ML delineation with almost no extra effort, with close results to the EDR method.

\section{Acknowledgements}

This study was partially supported by projects TEC2013-42140-R and TIN2014-53567-R from the MINECO with European Regional Development Fund (FEDER), Spain, and by Grupo Consolidado BSICoS (T96) from DGA (Aragón, Spain) and European Social Fund (EU). The last author was partially supported by CMUP (UID/MAT/00144/2013), which is funded by FCT (Portugal) with national (MEC) and European structural funds through the programs FEDER, under the partnership agreement PT2020.

\section{References}

[1] Brochard L, Martin GS, Blanch L, Pelosi P, Belda FJ, Jubran A, Gattinoni L, Mancebo J, Ranieri VM, Richard J-CM, et al., Clinical review: respiratory monitoring in the ICU -a consensus of 16. Crit. Care 2012;16(2):219.

[2] Yasuma F, Hayano J. Respiratory sinus arrhythmia: Why does the heartbeat synchronize with respiratory rhythm? Chest 2004; 125(2):683-90.

[3] Aström M, Santos HC, Sörnmo L, Laguna P, Wohlfart B. Vectorcardiographic loop alignment and the measurement of morphologic beat-to-beat variability in noisy signals. IEEE Trans. Biomed. Eng. 2000;47(4):497-506.

[4] Bailón R, Sörnmo L, Laguna P. ECG-derived respiratory frequency estimation. In F. Azuaje GD. Clifford and PE. McSharry, editors, Advanced methods and tools for ECG data analysis 2006, 215-244. Artech House, Inc.

[5] Cysarz, Zerm R, Bettermann H, Frtihwirth M, Moser M, Kroz M. Comparison of respiratory rates derived from heart rate variability, ECG amplitude, and nasal/oral airflow. Ann Biomed Eng. 2008; 36:2085-2094.

[6] Orphanidou C, Fleming S, Shah S, Tarassenko L. Data fusion for estimatig respiratory rate from a single-lead ECG. Biomed Signal Process Control 2013; 8:98-105.

[7] Mirmohamadsadeghi L, Vesin J.M. Respiratory rate estimation from the ECG using an instantaneous frequency tracking algorithm. Biomed Signal Process Control 2014; 14:66-72.

[8] Prudat Y, Vesin JM. Multi-signal extension of adaptive frequency tracking algorithms. Signal Process. 2009; 89:963973.

[9] Almeida R, Martínez JP, Rocha AP, Laguna P. Multilead ECG delineation using spatially projected leads from wavelet transform loops. IEEE Trans. Biomed. Eng., vol 56, no 8, 2009, pp 1996-2005.

[10] Noriega M, Martínez JP, Laguna P, Bail $\tilde{A}^{3} n$ R, Almeida R. Respiration Effect on Wavelet-Based ECG T-Wave End Delineation Strategies. IEEE Trans. Biomed. Eng 2012; 59:1818-1828.

[11] Noriega M, Marañón EJ, Pérez DR, Orini M, Almeida R. Respiratory rate estimation from multilead directions, based on ECG delineation, Proceedings of the 38th Annual International Conference of the IEEE Engineering in Medicine and Biology Society (EMBC16) in press

[12] Bolea J, Almeida R, Laguna P, Sörnmo L, Martínez JP. BioSigBrowser, biosignal processing interface. 9th International Conference on Information Technology and Applications in Biomedicine, ITAB 2009;1-4.

[13] Martínez JP, Almeida R, Olmos S, Rocha AP, Laguna P. Wavelet-based ECG delineator: evaluation on standard databases. IEEE Trans. Biomed. Eng. 2004;51:570-81.

[14] Van Huffel S, Vandewalle J. The Total Least Squares Problem: Computational Aspects and Analysisâ, ser. Frontiers in Applied Mathematics. Philadelphia: Society for Industrial and Applied Mathematics, 1991;9.

[15] Dower GE. The ECGD: a derivation of the ECG from VCG leads. Journal of Electrocardiol. 1984;17(2):189-91.

[16] Marple SL Digital Spectral Analysis. Englewood Cliffs, NJ, Prentice-Hall, 1987, Chapter7.

Address for correspondence:

Rute Almeida

Centro de Matemática da Universidade do Porto, Departamento de Matemática da FCUP, Rua do Campo Alegre, 687, 4169-007 Porto, Portugal

rbalmeid@fc.up.pt 